\title{
Modeling giant planets and brown dwarfs
}

\author{
Andreas Becker ${ }^{1}$, Nadine Nettelmann ${ }^{2}$, Ulrike Kramm ${ }^{1}$, \\ Winfried Lorenzen ${ }^{1}$, Martin French ${ }^{1}$ and Ronald Redmer ${ }^{1}$ \\ ${ }^{1}$ Institute of Physics, University of Rostock, \\ Universitätsplatz 3, 18051, Rostock, Germany \\ email: andreas.becker@uni-rostock.de \\ ${ }^{2}$ Dept. of Astronomy \& Astrophysics, University of California, \\ CA 95064 Santa Cruz, USA
}

\begin{abstract}
We present new results in modeling the interiors of Giant Planets (GP) and Brown Dwarfs (BD). In general models of the interior rely on equation of state data for planetary materials which have considerable uncertainties in the high-pressure domain. Our calculations are based on $a b$ initio equation of state (EOS) data for hydrogen, helium, hydrogen-helium mixtures and water as the representative of all heavier elements or ices using finite-temperature density functional theory molecular dynamics (FT-DFT-MD) simulations. We compare results for the BD Gliese 229B calculated with Saumon-Chabrier-Van Horn EOS (SCVH95) and our EOS data.
\end{abstract}

Keywords. equation of state, planetary systems, brown dwarfs

\section{Introduction}

We perform extensive electronic structure calculations for warm dense matter within finite-temperature density functional theory combined with molecular dynamics simulations for the ions (FT-DFT-MD) using the VASP code (Kresse \& Furthmüller 1996). We calculate EOS data for hydrogen (Holst et al. 2008), helium (Kietzmann et al. 2007), hydrogen-helium mixtures (Lorenzen et al. 2009) and water (French et al. 2009) within a temperature range up to $100,000 \mathrm{~K}$ and a pressure range up to $100 \mathrm{Mbar}$ as well as electrical conductivities, pair correlation functions and ion diffusion coefficients. We also determine the high-pressure phase diagrams and the location of metal-nonmetal transitions and of demixing regions.

With this input we model solar and extrasolar Giant Planets (GP) within standard three-layer structure models (Guillot 1999) using a mixture of our EOS, called LM-REOS (Linear Mixing-Rostock Equation Of State). Available observational constraints such as mass $M$, radius $R$, rotational period, gravitational moments $\mathrm{J}_{\mathrm{n}}$, the mean helium fraction $\overline{\mathrm{Y}}$ which is given by the solar value and the helium fraction at the surface $\mathrm{Y}_{1}$ are fulfilled by our models. Gravity data like $\mathrm{J}_{2}$ and $\mathrm{J}_{4}$ are used to fit metallicities, the size of the core, and the chemical composition within the layers.

Brown Dwarfs are assumed to be fully convective (Stevenson 1991) so that we use an adiabatic one-layer model for the calculations of their interior structure. In our computer code the radius is fitted to the mass by solving the differential equation for hydrostatic equilibrium and for mass conservation in a spherical shell for a given EOS and surface temperature. Typical central conditions for BD's are $\mathrm{p}_{\mathrm{c}} \propto 1000 \mathrm{Mbar}, \rho_{\mathrm{c}} \propto 10^{3} \mathrm{~g} / \mathrm{cm}^{3}$, and $\mathrm{T}_{\mathrm{c}} \propto 10^{6} \mathrm{~K}$. We apply our EOS tables down to $\sim 100 \mathrm{Mbar}$ and use the SaumonChabrier-Van Horn EOS (Saumon et al. 1995) for the deep interior. Smooth interpolation is performed between the different EOS tables. 


\section{Results}

We determine the isentrope of Saturn and find that it undergoes H-He demixing for a pressure larger than about 1 Mbar, see Lorenzen et al. (2009). This effect has probably a significant impact on its cooling time. We obtain very good agreement for the interior structure of Uranus and Neptune with predictions of dynamo models for their magnetic field made by Stanley \& Bloxham (2004) using the phase diagram of water, see Redmer et al. 2010. Application of our EOS data to Jupiter restricts the core mass to 1-5 Earth masses $\left(\mathrm{M}_{\mathrm{E}}\right)$ and the total mass of metals to 30-40 $\mathrm{M}_{\mathrm{E}}$, enriching the inner envelope over 5-9 times the solar value (Nettelmann et al. 2008). For the interior of the Neptune-like planet GJ $436 \mathrm{~b}$ we state that water occurs essentially in the plasma phase or in the superionic phase, but not in an ice phase, see Nettelmann et al. (2010).

For the Brown Dwarf Gliese 229B we obtain first models using the observational constraints given in Basri (2000): $R=1 R_{J u p}, M=30 M_{\text {Jup }}$ and $T_{\text {eff }}=1000 \mathrm{~K}$, see figure 1 . We assume that the BD neither has a core nor metals in the envelope. The lines show a difference for the central region in pressure, density and temperature of about $10 \%$ depending on the EOS. We obtain a radius $1.5 \%$ larger than $1 \mathrm{R}_{\mathrm{Jup}}$ applying LM-REOS and $5 \%$ applying SCVH95. Our aim for the future is a full description using ab initio EOS data such as LM-REOS.
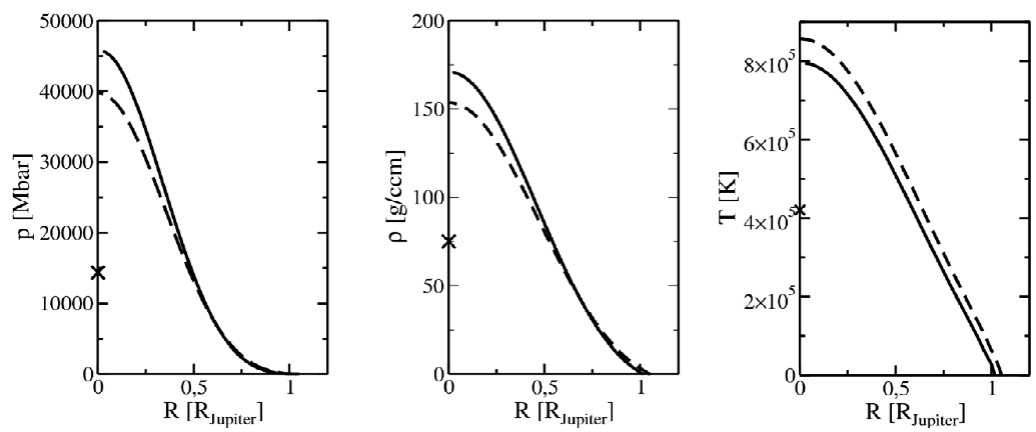

Figure 1. Profiles for pressure, density and temperature through Gliese 229B. Dashed-line profiles are calculated with SCVH95, solid lines with LM-REOS and SCvH95, the crosses represent central values given from Burrows \& Liebert (1993).

\section{References}

Basri, G. 2000, Scientific American, 05/2000, 27

Burrows, A. \& Liebert, J. 1993, Rev. Mod. Phys., 65, 301

French, M., Mattson, T. R., Nettelmann, N., \& Redmer, R. 2009, Phys. Rev. B, 79, 054107

Guillot, T. 1999, Science, 286, 27

Holst, B., Redmer, R., \& Desjarlais, M. P. 2008, Phys. Rev. B, 77, 184201

Kietzmann, A., Redmer, R., Desjarlais, M. P., \& Mattson, T. R. 2007, Phys. Rev. Lett., 98, 190602

Kresse, G. \& Furthmüller, J. 1996, Phys. Rev. B, 54, 11169

Lorenzen, W., Holst, B., \& Redmer, R. 2009, Phys. Rev. Lett., 102, 115701

Nettelmann, N., Holst, B., Kietzmann, A., French, M., Blaschke, D., \& Redmer, R. 2008, ApJ, 683,1217

Nettelmann, N., Kramm, U., Redmer, R., \& Neuhäuser, R. 2010, A\&A, 523, A26

Redmer, R., Mattson, T. R., Nettelmann, N., \& French, M. 2011, Icarus, 211, 798

Saumon, D., Chabrier, G., \& Van Horn, H. M. 1995, ApJS, 99, 713

Stanley, S. \& Bloxham, J. 2004, Nature, 428, 151

Stevenson, D. J. 1991, Annu. Rev. Astron. Astrophys. 29, 163 